\title{
Comparison of Implicit Schemes for the Incompressible Navier-Stokes Equations Stuart E. Rogers
}

Reprinted from

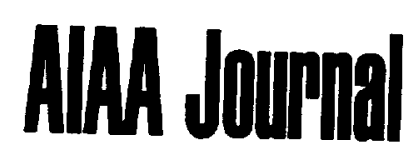

Volume 33, Number 11, Pages 2066-2072

\section{OALAA:}

A publication of the

American Institute of Aeronautics and Astronautics, Inc. 370 L'Entant Promenade, SW

Washington, DC 20024-2518 


\title{
Comparison of Implicit Schemes for the Incompressible Navier-Stokes Equations
}

\author{
Stuart E. Rogers* \\ NASA Ames Research Center, Moffett Field, California 94035-1000
}

\begin{abstract}
For a computational flow simulation tool to be useful in a design environment, it must be very robust and efficient. To develop such a tool for incompressible flow applications, a number of different implicit schemes are compared for several two-dimensional flow problems in the current study. The schemes include Point-Jacobi compared for several two-dine relaxation, incomplete lower-upper decomposition, and the generalized minimum relaxation, Gauss-Seidel tine relaxis residual method preconditioned with each of the three ols sclution for the laminar flow over a backward-facing terms of the computing time required to obtain a steady-state solution for the laminar fow over a backwards. The flow step, the flow over a NACA $\mathbf{4 1 1 2}$ airfoil, and the flow over a three-element airfoil using overset grids. The flow solver used in the study is the INS2D code that solves the incompressible Navier-Stokes equations that the generalized of artificial compressibility and upwind differencing of the convective terms. The results show that the generalions minimum residual method precon

methods by at least a factor of 2 .
\end{abstract}

\section{Introduction}

A LTHOUGH computational fluid dynamics (CFD) now has a lot to offer an engineer, there is still room for significant improvement. The efficiency and robustness of a flow solver are two of its most important features if it is to be successfully applied as a tool in the design process. This is especially true of Navier-Stokes methods, which require very fine resolution grids, particularly for high-Reynolds-number flows. When engineers need to study a number of design parameters, they often need to obtain hundreds of steady-state solutions to a particular problem. Use of optimization methods in design may require numerous flowfield solutions. In both these instances, a flow solver needs to produce solutions without requiring the users to tune the input numerical parameters for each computation that they run.

The goal of the current study is to determine an efficient algorithm for obtaining steady-state solutions to the incompressible NavierStakes solutions for two-dimensional flow problems. There is a wide range of applicability of such a flow solver in engineering fow analysis, including high-lift multi-element airfoil computations ${ }^{1}$ and propulsion flow analysis. ${ }^{2}$ These investigations are being performed for two-dimensional flows because these are cheaper-allowing the investigation of many parameters, schemes, and flow problemsand because a two-dimensional analysis tool can be quite valuable in its own right. This study will use the INS2D flow solver, ${ }^{3,4}$ and later the results can be utilized to improve the INS3D flow solver. ${ }^{5}$

There are many different types of solution techniques for steadystate incompressible Navier-Stokes computations, too numerous to discuss in detail here. The INS2D and INS3D flow solvers by default use an implicit Gauss-Seidel line-relaxation (LR) scheme. This scheme has performed quite well for a large number of different flow problems ${ }^{1-5}$ but has been shown to have some convergence problems for very fine meshes with multiple zones. For example, some fine-resolution, multizone grids used in recent multi-element airfoil calculations ${ }^{1}$ have taken several thousand iterations to converge, whereas most cases that are run with this flow solver converge within 200 iterations.

Received Dec 27 1994; presented as Paper 95-0567 at the AIAA 33rd Aerospace Sciences Meeting, Reno, NV, Jan. 9-12, 1995; revision received April 18. 1995; accepted for publication April 21, 1995. Copyright (C) 1995 April 18. 1995, accepted for punautics and Astronautics, Inc. No copyright by the Amencan Institute of Aeronander Title 17, U.S. Code. The U.S. Govis asserted in the United Sticense to exercise all rights under the copyright ernmed herein for Governmental purposes. All other rights are reserved by the copyright owner.

*Aerospace Engineer, Design Cycle Technologies Branch, Aeronautical Technological Division. Mail Stop T27B-1. Senior Member AIAA.
Recent work in iterative, Krylov-space matrix solvers ${ }^{6.7}$ has shown that some of these methods are applicable to CFD flow solvers. In particular, the generalized minimal residual (GMRES) method $^{8}$ is well suited to the matrix problems arising in implicit CFD solvers. There are two distinct ways to implement GMRES in a flow solver. One approach is to use GMRES to solve the linearized system of equations resulting from the application of a time-marching type of scheme, such as an implicit Euler scheme. The other formulation of scheme, such as an implicit Euler sches to directly solve the discrete form of the steady-state equations. The first approach has been attempted by many authors; see Refs. 9-11 for recent examples. The second approach was introduced by Wigton et al. ${ }^{12}$

The most important aspect of implementing GMRES is the preThe most important as stem of equations. For implementation in a CFD code, a good preconditioner is necessary for GMRES to converge. The current work investigates the use of GMRES to solve the linearized system of equations in delta form in the INS2D code. The code for the GMRES solver was obtained from the template software available as a companion to Ref. 7 . The GMRES method can be easily implemented so that any existing solution process can be utilized as a preconditioner. One commonly used preconditioner for the GMRES method is an incomplete lower-upper (ILU) factorization with zero additional fill. See Meijerink and van der Vorst ${ }^{13}$ for a discussion of ILU methods. The ILU solution scheme was added as an option to the INS2D code, along with a Point-Jacobi relaxation (PR) scheme, to go along with the Gauss-Seidel line-relaxation scheme already in the code. Presented in this paper are comparisons between six different solution processes: the three aforementioned algorithms run independently, and the GMRES method using these three algorithms as preconditioners, denoted as GMRES+ILU, GMRES+PR, and GMRES+LR.

In the following sections, the features of the INS2D flow solver are discussed, followed by a presentation of each of the implicit methods used in this study. The next section presents the computed results of each of these methods for three different flow problems: laminar flow over a backward-facing step, turbulent flow over a NACA 4412 airfoil, and turbulent flow over a three-element airfoil.

\section{Flow Solver}

The INS2D flow solver ${ }^{3.4}$ solves the Reynolds-averaged incompressible Navier-Stokes equations using the method of artificial compressibility, ${ }^{14}$ which adds a pseudotime derivative of pressure $p$ to the continuity equation, resulting in

$$
\frac{\partial p}{\partial \tau}=-\beta \nabla \cdot V
$$


where $\tau$ is the pseudotime, $V$ is the velocity vector, and $\beta$ is the artificial compressibility constant. This relaxes the elliptic nature of the equations and results in a hyperbolic-parabolic system. The solver is capable of solving both steady-state and time-dependent flow problems, although the current study is concerned only with steadystate solutions. The INS2D code is a finite difference, structuredgrid flow solver. It is capable of handling multiple-zone grids using either a patched multiblock (pointwise continuous) interface or an overlaid chimera interface between zones. The boundary conditions at the physical boundaries and at zonal boundaries are applied in an implicit fashion during the solution process. A third-order, upwinddifferencing scheme based on the method of Roe ${ }^{15}$ is used to discretize the convective terms, and the viscous terms are differenced using second-order central differences. The system of equations is integrated in pseudotime using an implicit Euler time discretization. The resulting discrete system of equations has the form

$$
\frac{Q^{n-1}-Q^{n}}{\Delta \tau}=-R^{n+1}
$$

where $Q$ is the vector of dependent variables (pressure, $u$ - and $v$-velocity components), $\Delta \tau$ is the time-step size in pseudotime, the superscript $n$ is the iteration number, and $R$ is the residual, comsystem is discrete form of the convective and viscous terms. This system is linearized about pseudotime-level $n$, resulting in

$$
\left(\frac{1}{\Delta \tau}+\frac{\partial R^{n}}{\partial Q}\right) \Delta Q=-R^{n}
$$

where $\Delta Q=Q^{n+1}-Q^{n}$. This equation is iterated until a steady state solution is obtained at which time $R^{n} \approx 0$. In the curren implementation, the Jacobian of $R$ on the left-hand side (LHS) of Eq. (3) is formed using a residual based on first-order differencing of the convective terms, whereas third-order differencing is used on the right-hand side (RHS). In addition, approximate Jacobians of flux differences from the upwind-differencing scheme are used, because exact Jacobians of these terms would require the formation of a tensor (see Ref. 16 for more details), and it has been assumed that the added computational costs would outweigh any benefit. The first-order LHS is used to reduce the bandwidth of the resulting LHS matrix, resulting in lower memory and computational requirements for the solution of Eq. (3). However, this use of approximate Jacobians can also slow the convergence to a steady state.

For turbulent flow calculations, the current study uses the turbu lence model of Baldwin and Barth. ${ }^{17}$ This model requires the solution of a single convective-diffusive partial-differential equation This equation is uncoupled from the mean-flow equations during the solution process. The convective terms in the turbulence model are discretized using a first-order upwind-differencing scheme. The resulting discrete equation for the turbulence model has the same form as Eq. (3), except $Q$ now represents a single variable at each grid point instead of three variables; the LHS of Eq. 3 is a banded matrix composed of five diagonals, each containing scalar entries. In all cases presented here, the turbulence model equation is solved using the same implicit scheme as the mean-flow equations.

\section{Implicit Schemes}

If Eq. (3) was solved exactly at each time step, and if the LHS of Eq. (3) was composed of the exact Jacobians, and an infinite time step was used, this would be a Newton iteration. In this case, quadratic convergence could be obtained if the $Q^{n}$ was close to the exact solution. Since the LHS is composed of an approximate Jacobian of the RHS. and since the turbulence model is uncoupled from the mean-flow equations, the current solution procedure is not a Newton iteration. Thus the goal is to obtain an approximate solution to Eq. (3) in an efficient manner. Several methods are used to attempt to do this. This discrete form of the matrix from the LHS of Eq. (3) is a pentadiagonal banded matrix, where each entry on the diagonal consists of $3 \times 3$ blocks. Equation (3) can be written as

$$
[D .0, \ldots, 0, A, B, C .0, \ldots, 0, E] \Delta Q=-R^{n}
$$

where $A, B, C, D$, and $E$ are the block diagonals. In the implementation of all of the implicit schemes, the code first computes and stores all of the terms in Eq. (4) and then proceeds with the solution procedure. This storage requires $48 N$ words, where $N$ is the total number of grid points. In the following, a subscript $i$ refers to a in a single vector index when one can consider all of the grid points . The subscripts $j$ and $k$ are indices in the data are stored in -space directions $\xi$ and $\eta$, respectively. When fastest changing (inner) index.

\section{ILU Factorization}

In the ILU formulation, the matrix on the LHS of Eq. (4) is replaced with the following factors:

$$
\begin{gathered}
{\left[D, 0, \ldots, 0, A, B^{\prime}\right]\left[B^{\prime}\right]^{-1}\left[B^{\prime}, C, 0, \ldots, 0, E\right]} \\
B_{i}^{\prime}=B_{i}-A_{i} C_{i-1}^{\prime}-D_{i} E_{i-j \max }^{\prime} \\
C_{i}^{\prime}=\left[B_{i}^{\prime}\right]^{-1} C_{i} \\
E_{i}^{\prime}=\left[B_{i}^{\prime}\right]^{-1} E_{i}
\end{gathered}
$$

Multiplying these factors together, one sees that a matrix of the same structure as the original LHS is obtained, except that there are additional diagonals of nonzero entries created just above the $D$ diagonal and just below the $E$ diagonal. These new entries are ignored in the approximation. This is known as ILU with zero additional fill, or ILU (0). See Ref. 12 for details on implementing ILU schemes with

The ILU solver requires some significant initialization work namely, the computation and storage of the $B^{\prime}$ diagonal. This requires $9 N$ extra storage locations. When used as a preconditioner, this is done once at the beginning of the GMRES solution process and used repeatedly during the GMRES iteration cycle. This new set of factors gives an easy system to solve. The solution process can be vectorized by setting up an inner loop operating on all points on a diagonal line defined by $j+k=$ constant.

\section{Point Relaxation}

The PR algorithm iteratively solves a block diagonal system formed by multiplying all of the nonmain block diagonals by the current estimate for $\Delta Q$ and moving this to the RHS. This is done for each point, sweeping sequentially through the mesh. A forward
sweep is composed of

$$
\begin{gathered}
{[B] \Delta Q^{\prime+1}=-R^{n}-[D, 0, \ldots, 0, A] \Delta Q^{\prime+1}} \\
-[C, 0, \ldots, 0, E] \Delta Q^{\prime}
\end{gathered}
$$

and a backward sweep is performed by solving

$$
\begin{aligned}
& {[B] \Delta Q^{\prime+1}=-R^{n}-[D, 0, \ldots, 0, A] \Delta Q^{\prime}} \\
& -[C, 0, \ldots, 0, E] \Delta Q^{\prime+1}
\end{aligned}
$$

In the current computations, a forward sweep plus a backward sweep counts as one sweep, denoted as PR(1). The solution process is initialized by setting $\Delta Q$ to zero. Then, a lower-upper (LU) factorto solve this equation is formed. Thus the number of operations process. This equation is minimized during the repeated sweeping to compute $\Delta Q$ for all be vectorized by setting up an inner loop given by $j+k=$ constant.

\section{Line Relaxation}

The LR process is similar to the PR method, except that more terms are kept on the LHS and a block tridiagonal system of equaimplemented so that entire grid line at once. The algorithm is to be the sweep direction. For solving direction can be selected sweep is composed of

$$
[A, B, C] \Delta Q^{\prime+1}=-R^{n}-[D] \Delta Q^{l+1}-[E] \Delta Q^{\prime}
$$

and a backward sweep is performed by solving

$$
[A, B, C] \Delta Q^{\prime+1}=-R^{n}-[D] \Delta Q^{\prime}-[E] \Delta Q^{\prime+1}
$$


A forward plus a backward sweep counts as two sweeps and is denoted by $L R(2)$. This process is also initialized by setting $\Delta Q$ to zero. Then, an $\mathrm{LU}$ factorization of the tridiagonal system is formed to minimize the number of operations during the sweeping process. The sweeping process is recursive and cannot be vectorized. It should be noted that the LR and PR schemes each require the same number of operations per point per sweep, because whereas the PR only has to solve a block diagonal system instead of a block tridiagonal system, it has additional block vector multiply operations for he additional RHS terms. The PR scheme will run faster on a vector computer because it can be vectorized. In practice, the PR routine runs about twice as fast as the LR routine on a Cray $C-90$ computer.

In both the PR and LR algorithms, zonal boundary conditions are enforced during the sweeping process. Typically, multiple sweeps are performed at each iteration. When computing a multiple zone grid, all zones are swept once before moving onto a second sweep. After each zone is swept this new $\Delta Q^{\prime+1}$ is passed to all other zones that use this zone for their boundary conditions. In this fashion. information is propagated across zonal boundaries implicitly.

\section{GMRES Method}

The GMRES procedure of Saad and Schultz ${ }^{8}$ is an iterative procedure for solving the linear system of equations of the form

$$
M x-b=0
$$

or. in the left preconditioned form,

$$
P M x-P b=0
$$

where $P$ is the preconditioner that is an approximation to $M^{-1}$. The preconditioned matrix $P M$ will have a smaller spectral radius than $M$. resulting in faster convergence for the GMRES procedure. A GMRES procedure using $k$ search directions, known as GMRES $(k)$, forms an approximation to the solution vector $x$ given by

$$
x_{k}=x_{10}+y_{1} v_{1}+\cdots+y_{k} v_{k}
$$

where $x_{i}$ is an initial guess to the solution $x$. The $v_{i}$ are orthonormal vectors formed from the process

$$
r_{0}=P M x_{1}-P b . \quad v_{1}=r_{0} /\left\|r_{0}\right\|
$$

lierate: for $j=1,2, \ldots k$ :

$$
\begin{gathered}
h_{i, j}=\left(P M v_{j}, v_{i}\right), \quad i=1,2, \ldots, j \\
w_{j+1}=P M v_{j}-h_{1 . j} v_{1}-h_{2, j} v_{2}-\cdots-h_{j, j} v_{j} \\
h_{j+1 . j}=\left\|w_{j+1}\right\| \\
v_{j+1}=w_{j+1} /\left\|w_{j+1}\right\|
\end{gathered}
$$

The $v_{i}$ coefficients are computed so that the norm of the residual $\left\|M x_{4}-b\right\|$ is minimized. An estimate of this norm is available ing solution process as a function of the $h_{i, j}$ The process requires approximately $(4+k)^{*} 3^{*} N$ words variables. The process requires aprow equations; therefore, it is not of memory to apply to the mean-flow equation this memory usage, practical to use large values of $k$. Because of this memory in the current work $k$ is limited to no more than $k=$ mation process to capability of the GMRES algorithm allows the iteration process to continue beyond $k=10$ in this case. This is done simply by setting $x_{11}=x_{11}$ and restarting from the beginning. If a total of 30 GMRES search directions is specified to the code, then two restarts are used. This is designated as GMRES(30).

MRES algorithm is implemented here so that the iterations

The GMRES algorithm imp of two criteria. The first is simply can be stopped based on either of two criteria. The first to be used to specify the maximum number of search directions to be used The second is to specify a tolerance for the error. Of the norm of the GMRES process is that an accurate estimate of the norm of the residual is computed as part of the solution process. Using (in terms of tests. it was found that it was generally more efficient (in terms of
obtaining a converged steady-state flow solution) to specify a set number of search directions for GMRES than it was to specify a tolerance for the norm of the residual.
Because the preconditioner must be utilized once for each GMection, it needs to be relatively cheap. Thus, when RES search direction, it need used a preconditioner for GMRES, the PR and LR sche the relaxation process are used.

\section{Computed Results}

different methods has been tested for three different Each of the different methods hackward-facing step, turbulent geometries: laminar flow over a backwarbulent flow over a threeflow over a NACA 4412 airfoil, and and each method, calculations element airfoil. For each geomal ime-step size, an optimal value were run to determine an optimal telaxation sweeps or GMRES for $\beta$, and an optimal number calculations determined the best possible search directions. These calculations determin case; then the most performance of each method for a particular case, the best method efficient run of each method was used to determined the best mich for each case. Most cases ran best using an infite tis on is implemented by setting $\Delta \tau=10^{12}$, so that $1 / \Delta \tau$ is of machine zero. In some cases, this large In other cases, a large instability, and the time step was reduced. In other resulted in better time step remained stable, but a smaller times usually involved running efficiency. Testing different time-step sizest different values of $\beta$, with $\Delta \tau=10^{12}, 10.0,1.0$, and 0.1 . To test $5,50,100$, and 200 . See cases were run using values of $0.1,1,5,10$ effect of $\beta$ on the convergence. Ref. 5 for an example showing the effect of $\beta$ on the colocity is plot-

In each comparison, the maximum divergence of velocit (CPU) sected vs computing time, given in central processing unols are overlaid onds on a Cray C-90 computer. In addition, sym the number of on the plotting line at every 50 iterad. Most cases are run until the iterations required by each method. Most cases are run und, a useful divergence of velocity reaches machine zero. In general this value is steady-state solution has been obtained long maximum nondimenreached; usually this requires only that the maximum notely $10^{-2}$. sional divergence of velocity be reduced to approximmarizing the Finally, for each computational test case, a table summant The folconvergence characteristics for each scheme is present the value of $\beta$ and $\Delta \tau$ lowing parameters are given in thector, or average factor by which used in the run; the amplificed ach iteration; the computing time used the residual is reduced at each iterations per point per iteration, given in microse the solution divided by the time in milliseconds used to converge the solution, the solution was number of grid points. To generate these num nondimensional diverconsidered converged when the maximum nondithe amplification gence of velocity dropped below $10^{-3}$. Although the amplifich charfactor and computing time per point per iteration are useful che the acteristics of an algorithm, they do not gis a the best measure of the efficiency; the last column in these table
overall performance of the algorithm.

\section{Backward-Facing Step}

The laminar flow over a backward-facing step was computed for an expansion ratio of 1101.94 . The Reynolds number, based on the downstream height and the average inflow velocity, was $8 \times 10^{2}$. Figure 1 shows a close view of the grid near the step and the specified inflow velocity vectors. The inflow was 2 step heights upstream of the step, and the outflow boundary was placed 50 step heights downstream of the step. A single-zone $\mathrm{H}$ grid with dimensions of $160 \times 61$ was used. The grid points inside the step were blanked out, and the no-slip boundary conditions for the step faces were applied to internal grid points. This flow problem has been used previously as a validation $\operatorname{case}^{3}$ for the INS2D flow solver. These previous results showed good agreement with the experimental results of Armaly et al. ${ }^{18}$ The Reynolds number $8 \times 10^{2}$ case was the highest Reynolds number nu in this previous study and was the slowest to converge.

For the backward-facing step flow, the only schemes that did not un best with an infinite time step were the ILU scheme, which was unstable for $\Delta \tau>1.0$, and GMRES +LR, which was more

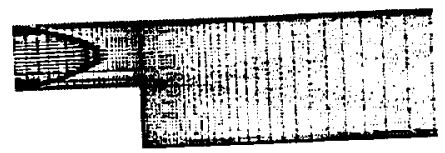

Fig. 1 Grid and inflow velocity vectors for the backwardfacing step flow. 


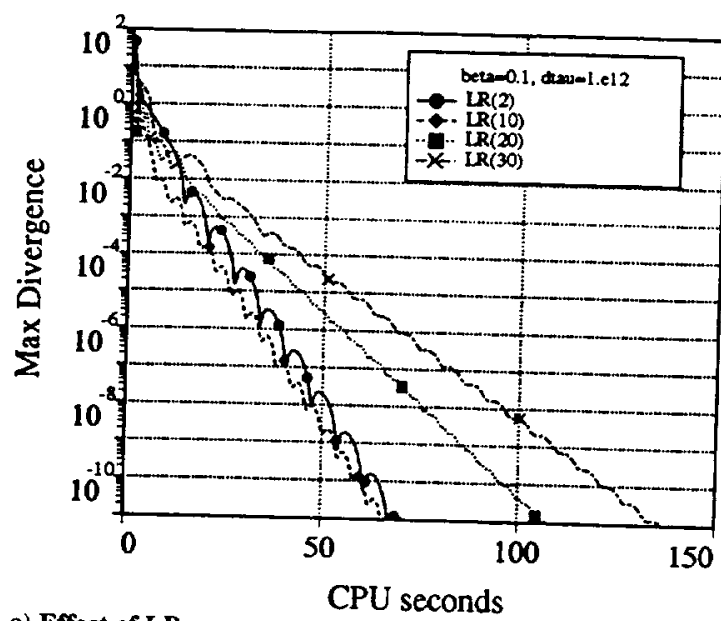

a) Effect of LR sweeps

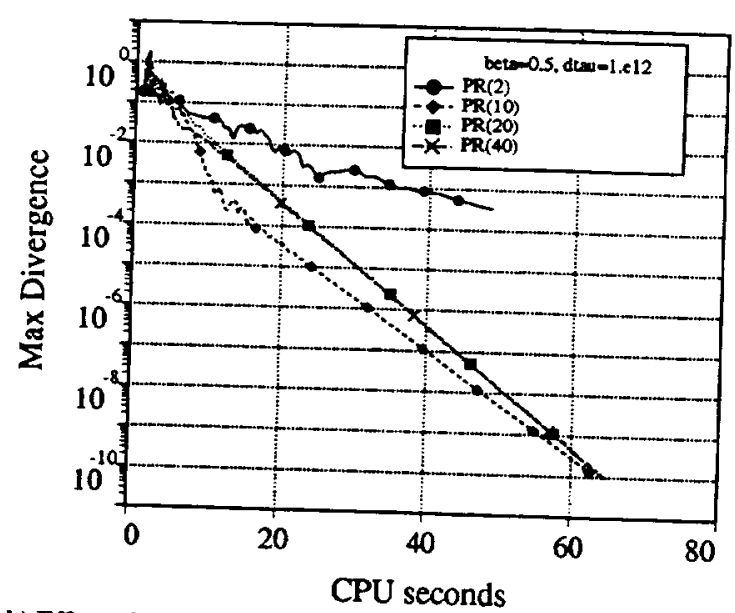

b) Effect of PR sweeps

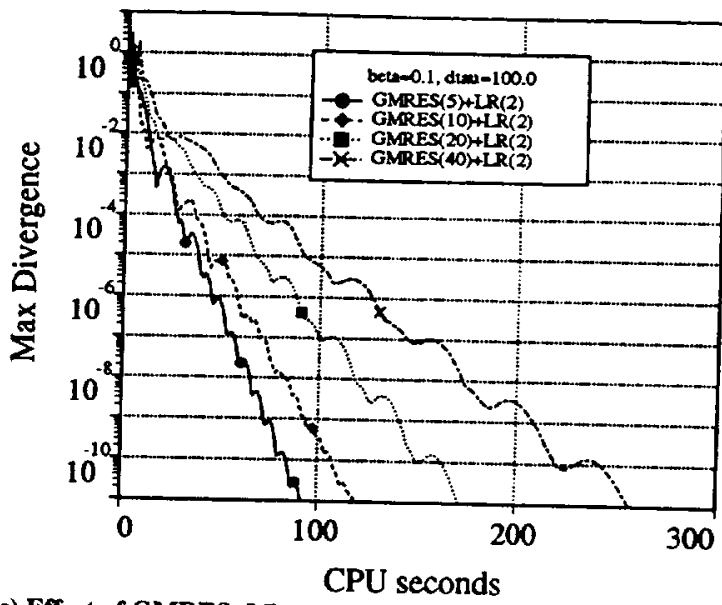

c) Effect of GMRES+LR search directions

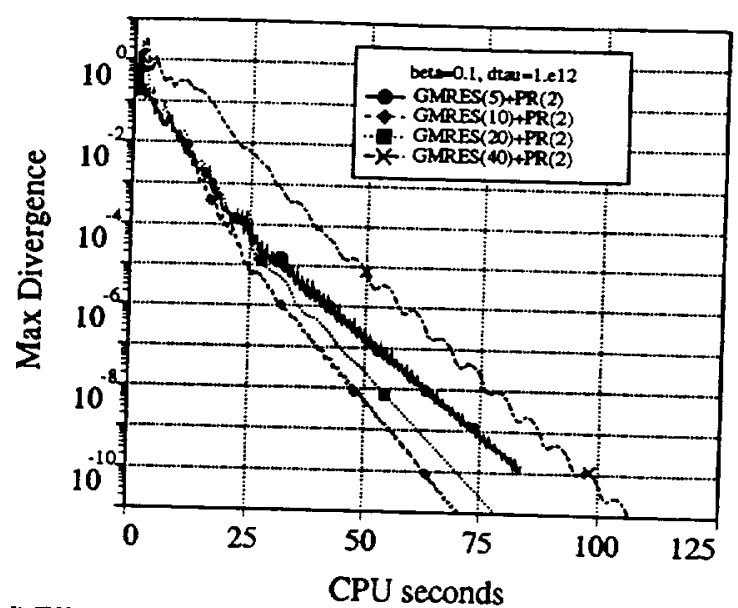

d) Effect of GMRES+PR search directions

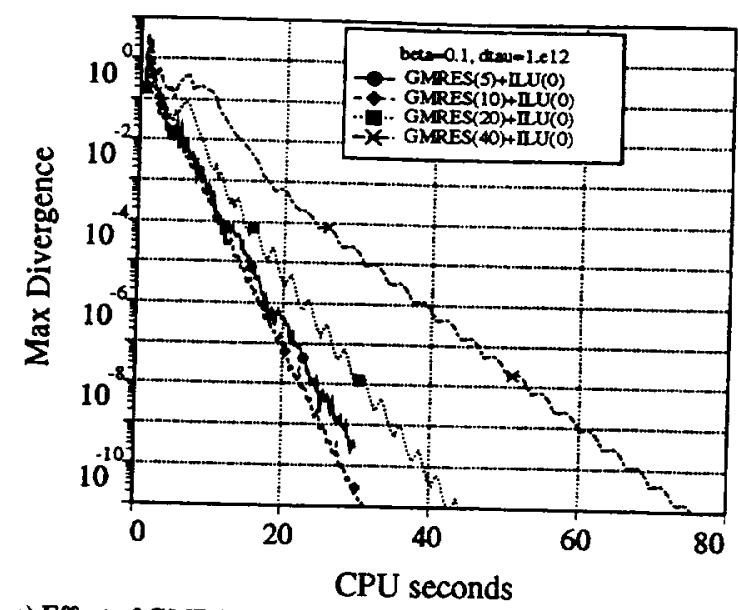

e) Effect of GMRES+ILU search directions

Fig. 2 Convergence for the backward-facing step flow.

efficient with $\Delta \tau=100$. Figure 2 shows the convergence for each scheme for a different number of sweeps/search directions. The LR scheme is best when using only 10 sweeps; the efficiency of the PR scheme is nearly constant as long as it is using at least 10 sweeps. The GMRES schemes are most efficient using either 5 or 10 search directions. For all of these schemes, it can be seen that when more sweeps/search directions are used they converge in fewer iterations, but the penalty of extra computing time per iteration causes this approach to be less efficient. Figure 3 shows the best convergence plot for each method for the backward-facing step flow; a summary of the convergence properties is shown in Table 1. Figure 3 and the last column of Table 1 show that GMRES + ILU converges faster than al other methods; the PR method is not far behind. The GMRES+LR scheme is less efficient than the LR scheme alone, even though it converges in significantly fewer iterations. The LR scheme shows the lowest amplification factor, but at nearly double the cost per iteration as the GMRES+ILU method and thus requires more time to converge.

\section{NACA 4412 Airfoil}

The flow over a NACA 4412 airfoil at a Reynolds number of $1.5 \times 10^{6}$ and an angle of attack of $13.87 \mathrm{deg}$ was computed using 
Table 1 Convergence for backward-facing step

\begin{tabular}{lccccc}
\hline Scheme & $\beta$ & $\Delta \tau$ & A.F. & $\begin{array}{c}\text { Microseconds/ } \\
\text { poind/iteration }\end{array}$ & $\begin{array}{c}\text { Milliseconds/ } \\
\text { point }\end{array}$ \\
\hline LR(10) & 0.1 & $10^{12}$ & 0.818 & 40.1 & 2.17 \\
PR(10) & 0.5 & $10^{12}$ & 0.912 & 16.7 & 1.69 \\
ILU & 0.1 & 1 & 0.994 & 9.9 & 23.00 \\
GMRES(5)+LR & 0.1 & 100 & 0.788 & 61.4 & 2.70 \\
GMRES(10)+PR & 0.1 & $10^{12}$ & 0.850 & 32.9 & 1.97 \\
GMRES(10)+ILU & 0.1 & $10^{12}$ & 0.826 & 21.1 & 1.14 \\
\hline
\end{tabular}

Table 2 Convergence for NACA 4412 grid 1

\begin{tabular}{lccccc}
\hline Scheme & $\beta$ & $\Delta \tau$ & A.F. & $\begin{array}{c}\text { Microseconds/ } \\
\text { point/iteration }\end{array}$ & $\begin{array}{c}\text { Milliseconds/ } \\
\text { point }\end{array}$ \\
\hline LR(5) & 10 & $10^{12}$ & 0.862 & 17.8 & 1.98 \\
PR(20) & 10 & $10^{12}$ & 0.785 & 34.8 & 2.31 \\
ILU & 20 & $10^{12}$ & 0.959 & 9.1 & 3.55 \\
GMRES(10)+LR & 5 & $10^{12}$ & 0.680 & 73.8 & 3.17 \\
GMRES(10)+PR & 5 & $10^{12}$ & 0.682 & 49.3 & 2.12 \\
GMRES(10)+ILU & 5 & $10^{12}$ & 0.676 & 27.2 & 1.14 \\
\hline
\end{tabular}

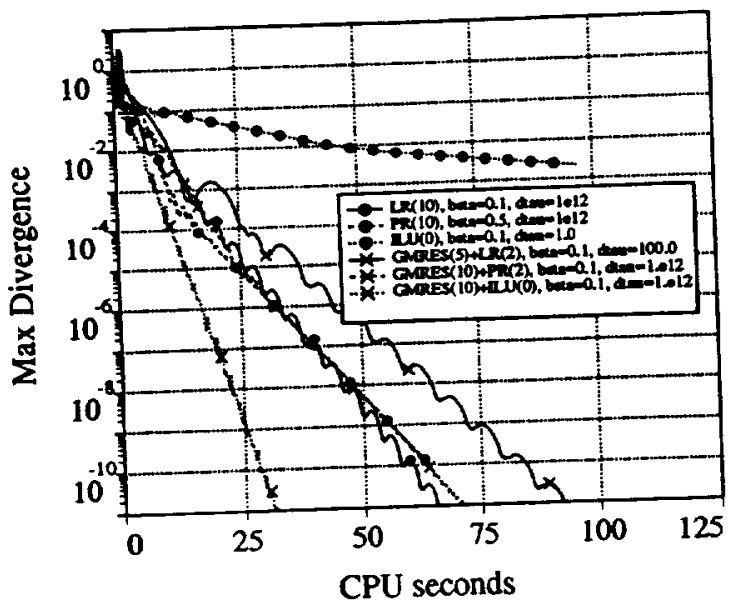

Fig. 3 Summary of convergence for the backward-facing step flow.

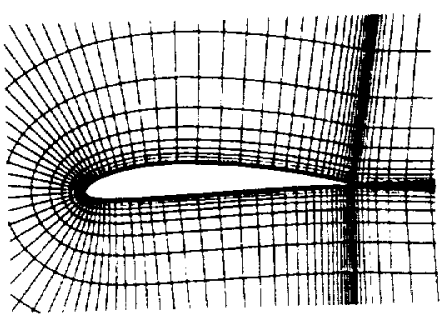

Fig. $4119 \times 31$ grid around the NACA 4412 airfoil.

four grids of varying refinement. The dimensions of these grids are $119 \times 31,237 \times 61,473 \times 121$ and $945 \times 241$. These will be referred to as grids $1,2,3$, and 4 , respectively. Grids 1,2 , and 3 were generated by removing every other point in each direction from the succeeding finer grid. The normal spacing on the finest grid was set to $5 \times 10^{-6}$ chords. Figure 4 shows the airfoil configuration with grid 1 . This configuration has been studied experimentally by Coles and Wadcock ${ }^{19}$ Previous computations with the INS2D code $e^{20}$ showed good agreement with the experimental data. At this angle of attack the airfoil is near stall conditions and has a amgle trailing edge.

In the computations using grid 1 , all of the methods ran with a time step of $10^{12}$. It was found that the LR scheme was most efficient using five sweeps. The PR runs converged well for 10,20 , and 40 sweeps, with 20 being the best. The GMRES schemes showed a loss in efficiency when using more than 10 search directions. Figure 5 plots the benterence of each method. This figure shows similar trends 作 to the backward
Table 3 Convergence for NACA 4412 grid 2

\begin{tabular}{lccccc}
\hline Scheme & $\beta$ & $\Delta \tau$ & A.F. & $\begin{array}{c}\text { Microseconds/ } \\
\text { pointiteration }\end{array}$ & $\begin{array}{c}\text { Milliseconds/ } \\
\text { point }\end{array}$ \\
\hline LR(10) & 50 & $10^{12}$ & 0.888 & 25.2 & 3.68 \\
PR(20) & 50 & $10^{12}$ & 0.914 & 26.6 & 5.13 \\
ILU & 10 & 0.1 & 0.990 & 6.7 & 12.00 \\
GMRES(5)+LR & 5 & $10^{12}$ & 0.827 & 40.5 & 3.77 \\
GMRES(10)+PR & 50 & 1.0 & 0.865 & 37.4 & 4.48 \\
GMRES(10)+ILU & 5 & $10^{12}$ & 0.780 & 19.6 & 1.45 \\
\hline
\end{tabular}

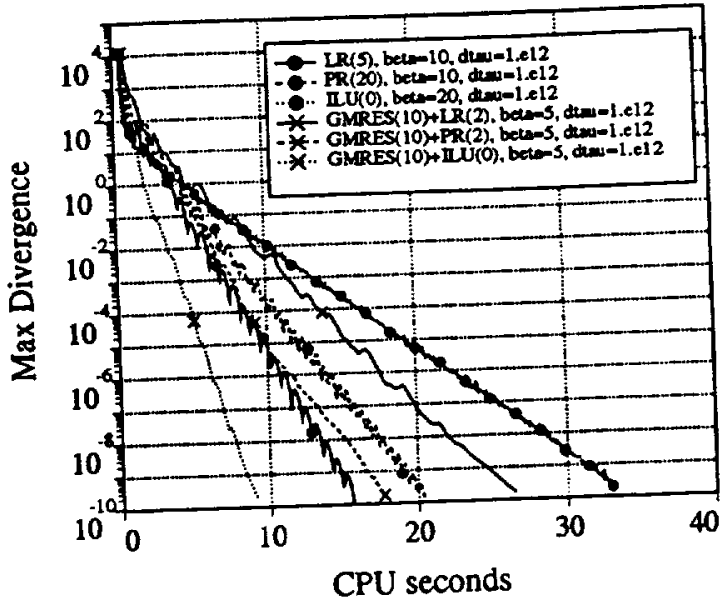

Fig. 5 Convergence for the fiow over a NACA 4412 airfoil with grid 1.

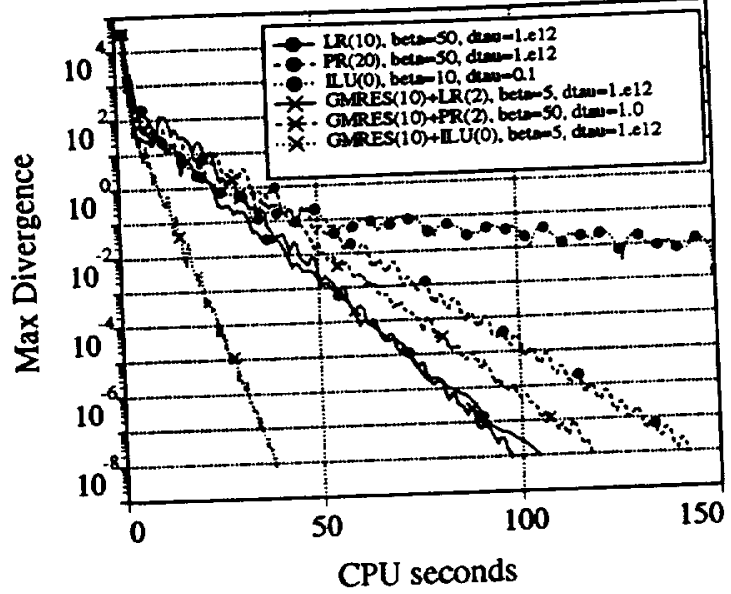

Fig. 6 Convergence for the flow over a NACA 4412 airfoil with grid 2.

had remarkably low amplification factors, below 0.7 , for this case. All methods had higher cost per point per iteration for this case because the smaller dimensions led to shorter vector lengths. The cost per point to converge for the GMRES+ILU was the same as the laminar backward-facing step flow.

The results for grid level 2 are shown in Fig. 6 and in Table 3 . or this grid, the GMRES+ILU approach outperforms all of the ther methods by at least a factor of 2 . In all cases, the amplification factor and the cost per point have increased with the increase in grid density.

Based on the results thus far, it is apparent that $L R$ and $P R$ are too expensive to be used as effective preconditioners for GMRES. It was also found that the ILU scheme alone cannot handle finer grid cases, as evidenced by the decay of the ILU amplification factor to 09 for the previous case. Thus, for the remainder of test cases in 0.99 for the previous case. Thus, for the rem eliminated. The results this study, these three approacho for the grid 3 case are shown of the three remaining methods for the grid 3 case are shown in Fig. 7 and Table 4. All the 1.0. Again, the GMRES +ILU

Computations for grid level 4 showed that only the GMRES+ILU Come results of this run are 
Table 4 Convergence for NACA 4412 grid 3

\begin{tabular}{lccccc}
\hline \hline Scheme & $\beta$ & $\Delta \tau$ & A.F. & $\begin{array}{c}\text { Microseconds/ } \\
\text { pointiteration }\end{array}$ & $\begin{array}{c}\text { Milliseconds' } \\
\text { point }\end{array}$ \\
\hline LR(10) & 100 & 1.0 & 0.953 & 23.2 & 8.79 \\
PR(40) & 5 & 1.0 & 0.929 & 51.3 & 12.56 \\
GMRES(10)+ILU & 5 & 1.0 & 0.917 & 18.7 & 3.91 \\
\hline
\end{tabular}

Table 5 Convergence for NACA 4412 grid 4

\begin{tabular}{lccccc}
\hline \hline Scheme & $\beta$ & $\Delta \tau$ & A.F. & $\begin{array}{c}\text { Microseconds/ } \\
\text { pointiteration }\end{array}$ & $\begin{array}{c}\text { Milliseconds/ } \\
\text { point }\end{array}$ \\
\hline GMRES(10)+ILU & 5 & 1.0 & 0.961 & 23.1 & 11.2 \\
\hline \hline
\end{tabular}

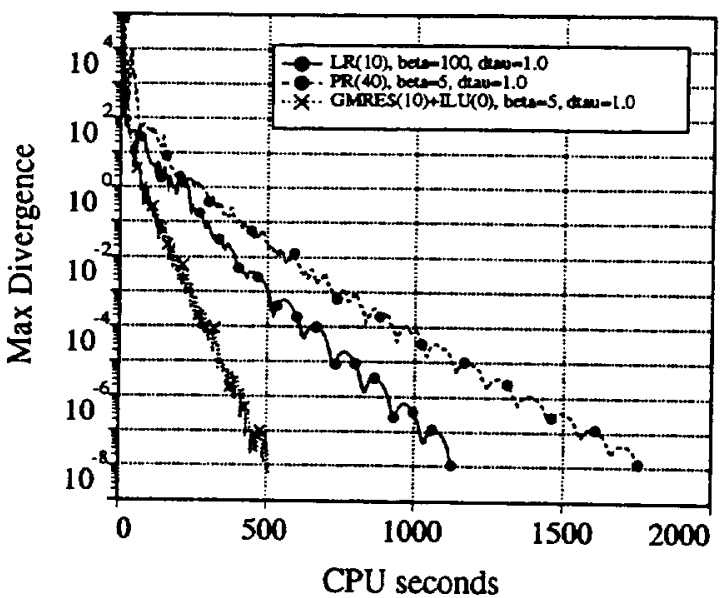

Fig. 7 Convergence for the flow over a NACA $\mathbf{4 4 1 2}$ airfoil with grid 3.

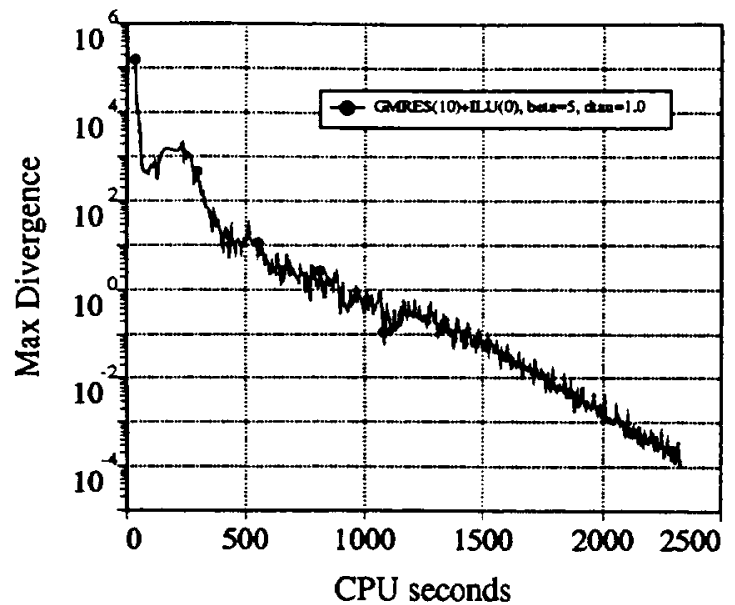

Fig. 8 Convergence for the flow over a NACA 4412 airfoil with grid 4.

shown in Fig. 8 and Table 5. Examining the trend of the convergence rate over all four grid levels shows that all methods suffered an increase in the amplification factor with increasing density. The effect of this is shown in Fig. 9, which plots the CPU time required to reach convergence per point vs the grid level for all of the airfoil computations. Even the most robust method, GMRES+ILU, saw an order-of-magnitude increase in this CPU cost from the coarsest grid to the finest grid. All of the methods required a reduction in the timestep size as the grid levels increased. Increasing the $\beta$ parameter tended to be a stabilizing influence; the LR and PR methods tended to require larger $\beta$ for the finer grids. One apparent contradiction to this is the use of $\beta=5$ for the PR grid 3 case. In fact, the PR convergence for grid 3 did not vary greatly with $\beta$; values from 5 to 100 all converged slowly. The $\beta=5$ case was slightly better than the $\beta=100$ run. One distinct advantage of the GMRES+ILU method is that it has optimum convergence with the same value of $\beta$ for all of the grid levels.
Table 6 Convergence for three-element arifoil

\begin{tabular}{lccccc}
\hline \hline Scheme & $\beta$ & $\Delta \tau$ & A.F. & $\begin{array}{c}\text { Microseconds/ } \\
\text { point/iteration }\end{array}$ & $\begin{array}{c}\text { Milliseconds' } \\
\text { point }\end{array}$ \\
\hline LR(10) & 100 & $1.0^{2}$ & 0.990 & 22.6 & 49.7 \\
PR(20) & 10 & 1.0 & 0.927 & 52.8 & 14.7 \\
GMR(10)+ILU & 10 & $10^{12}$ & 0.892 & 29.2 & 5.37 \\
\hline
\end{tabular}

${ }^{a} \Delta \tau$ reduced by a factor of 0.5 every 250 iterations

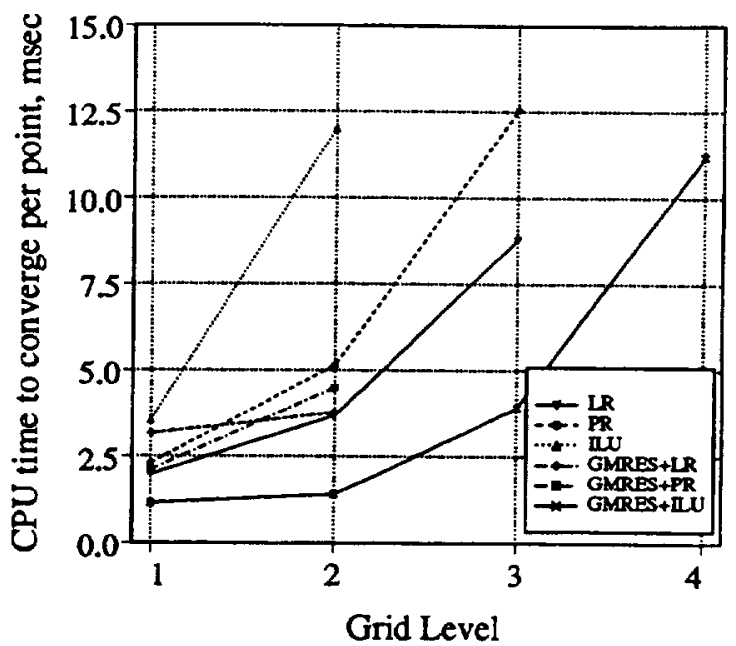

Fig. 9 CPU time to converge per point vs grid level for the fiow over a NACA 4412 airfoil.

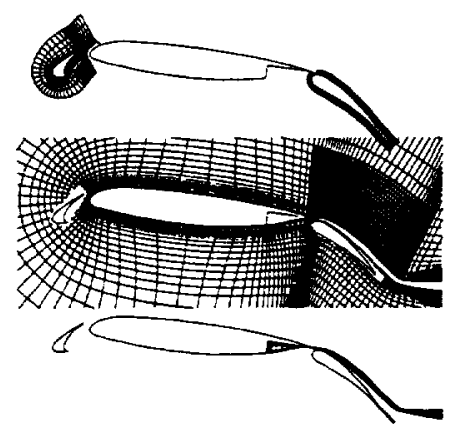

Fig. 10 Grid around the three-element airfoil.

\section{Three-Element Airfoil}

The flow over a three-element airfoil was computed at an angle of attack of $8.0 \mathrm{deg}$ and a Reynolds number of $9 \times 10^{6}$. This geometry has recently become a standard test case for multielement airfoil flows. This geometry is a McDonnell Douglas airfoil and has been tested extensively at the NASA Langley low-turbulence pressure tunnel (LTPT). ${ }^{21}$ The configuration consists of a leading-edge slat deflected $30 \mathrm{deg}$ and a trailing-edge flap deflected $30 \mathrm{deg}$. This geometry has been used as a test case for the INS2D flow solver for a number of turbulence model and grid resolution studies.' The grid and flow conditions of the current problem were some of the most difficult cases to converge in the previous study.

Figure 10 shows the grids used for the three-element airfoil. For clarity, only every other grid line in each direction is shown. A total of 68,000 grid points and six zones were used: a $121 \times 41 \mathrm{C}$ grid around the slat (top of Fig. 10); a $321 \times 101 \mathrm{C}$ grid around the main element (near field shown in middle of Fig. 10); a $141 \times 51 \mathrm{C}$ grid around the flap (top of Fig. 10); a $41 \times 31 \mathrm{H}$ grid in the wake of the flap (bottom of Fig. 10); a $131 \times 61 \mathrm{H}$ grid extending from the main elements' flap cove to the downstream far field (bottom of Fig. 10); and a $141 \times 101$ embedded grid above the flap, used to help resolve the merging wake in this region (middle of Fig. 10). The normal wall spacing for all grids is $2 \times 10^{-6}$ chords. The overlaid chimera scheme allows individual grids to be generated for each airfoil element. When the grid for one element intersects another airfoil element, a hole is cut to remove grid points lying inside the element. This creates a hole boundary. The fringe-point variables on the hole boundaries are updated by interpolating the value of 


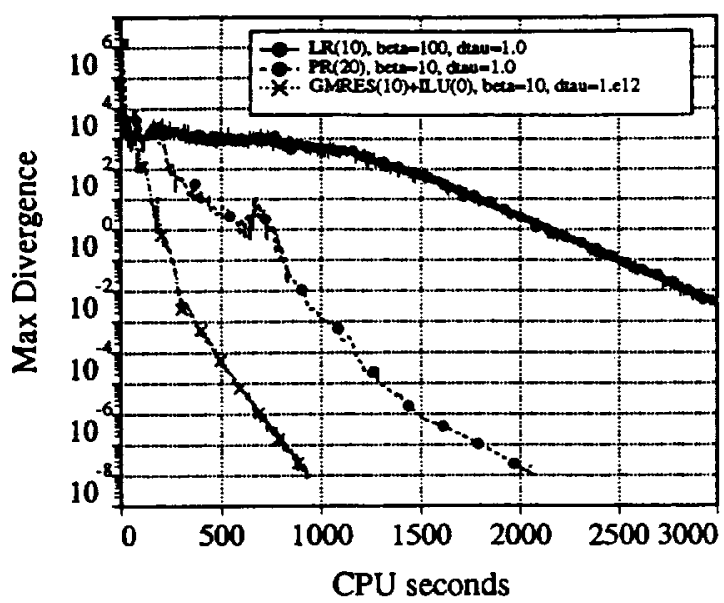

Fig. 11 Summary of convergence for the flow over a three-element airfoil.

the dependent variables from interior points of neighboring grids. Similarly, the variables on the outer boundaries of all but the mainelement grid are updated using interpolation of dependent variables from neighboring grids.

The LR and PR computations for this configuration required a reduction in the time step to $\Delta \tau=1.0$ to remain stable; GMRES+ILU ran with a time step of $10^{12}$. The LR computations were extremely slow to converge and were found to benefit from a further decrease in the time step during the calculations, and so the LR time step was decreased by a factor of 2 every 250 iterations. The PR computations did not suffer from the same problems. It was found that the PR computations converged best using 20 sweeps. The GMRES+ILU computations performed best with 10 search directions. The best performance of each method is plotted in Fig. 11 and shown in Table 6. It can be seen that the GMRES + ILU method outperformed the other two; it converges about nine times faster than the LR scheme and about three times faster than the PR computations.

\section{Conclusion}

Several implicit schemes have been implemented into the INS2D code and tested for the flow over a backward-facing step, a NACA airfoil, and a three-element airfoil. The results indicate that when running on a single processor the GMRES method preconditioned with ILU factorization outperforms line relaxation and point relaxation and that the latter two methods are not efficient preconditioners for GMRES. The GMRES+ILU method has provided between a factor of 2 and 9 improvement in CPU costs over previously published results of the INS2D code. In addition, the point-relaxation scheme performed remarkably well for the three-element airfoil problem. Extensive tests have indicated that GMRES is most efficient when using 10 search directions; if more search directions are used, the computation will converge in fewer iterations, but at a greater cost. The GMRES+ILU algorithm also remained more stable than the LR or PR methods; it ran with larger time steps and did not require an increase in the value of the artificial compressibility constant $\beta$ for the finger grids used in the NACA 4412 airfoil calculations.

The GMRES+ILU algorithm has been shown to work quite well for a two-dimensional flow solver. The success with this algorithm has also been observed recently by others. ${ }^{6,9-11}$ In particular, Venkatakrishnan and Mavriplis ${ }^{11}$ performed a similar study of implicit solvers for an unstructured flow code; they found their GMRES+ILU worked best. As it was utilized here, the GMRES algorithm would be very memory intensive for a three-dimensional flow solver. Future work will concentrate on utilizing a matrix-free method of the GMRES algorithm. The most important part of this work will be determining an effective preconditioner that will not require significant amounts of memory.

\section{References}

'Rogers, S. E., Menter, F. R., Mansour, N. N., and Durbin, P. A., "A Comparison of Turbulence Models in Computing Multi-Element Airfoil Flows," AIAA Paper 94-0291, Jan. 1994.

${ }^{2}$ Kiris, C., Kwak, D., and Rogers, S. E., "Computation of Incompressible Viscous Flows Through Turbopump Components," NASA TM-103911. March 1992

${ }^{3}$ Rogers, S. E., and Kwak, D., "An Upwind Differencing Scheme for the Steady-State Incompressible Navier-Stokes Equations," Journal of Applied Numerical Mathematics, Vol. 8, No. 1, 1991, pp. 43-64.

${ }^{4}$ Rogers, S. E., and Kwak, D., "An Upwind Differencing Scheme for the Time Accurate Incompressible Navier-Stokes Equations," AIAA Paper 882583, June 1988; see also AIAA Journal, Vol 28, No. 2, 1990, pp. 253-262.

${ }^{5}$ Rogers, S. E., Kwak, D., and Kiris, C., "Numerical Solution of the Incompressible Navier-Stokes Equations for Steady-State and Time-Dependent Problems," AIAA Paper 89-0463, Jan. 1989; see also AIAA Journal, Vol. 29. No. 4, 1991, pp. 603-610.

${ }^{6}$ Saad, Y., "Supercomputer Implementations of Preconditioned Krylov Subspace Methods," Algorithmic Trends in Computational Fluid Dynamics, edited by M. Y. Hussaini, A. Kumar, and M. D. Salas, Springer-Verlag, New York, 1993, pp. 107-136.

${ }^{7}$ Barrett, R., Berry, M., Chan, T., Demmel, J., Donato, J., Dongarra, J., Eijkhout, V., Pozo, R., Romine, C., and van der Vorst, H., Templates for the Solution of Linear Systems: Building Blocks for Iterative Methods, unpublished book available on NETLIB: ftp netlib2.cs.utk.edu; cd linalg; get templates.ps; 1993.

${ }^{8}$ Saad, Y., and Schultz, M. H., "GMRES: A Generalized Minimal Residual Algorithm for Solving Nonsymmetric Linear Systems," SIAM Journal of Scientific and Statistical Computing, Vol. 7, No. 3, 1986, pp. 856-869.

${ }^{9}$ Barth, T. J., and Linton, S. W. "An Unstructured Mesh Newton Solver for Compressible Fluid Flow and Its Parallel Implementation," AIAA Paper 95-0221, Jan. 1995.

${ }^{10}$ Ajmani, K., Liou, M., and Dyson, R. W., "Preconditioned Implicit Solvers for the Navier-Stokes Equations on Distributed-Memory Machines," AIAA Paper 94-0408, Jan. 1994.

${ }^{11}$ Venkatakrishnan, V., and Mavriplis, D. J., "Implicit Solvers for Unstructured Meshes," Journal of Computational Physics, Vol. 105, No. 1. 1993. pp. 83-91.

${ }^{12}$ Wigton, L. B., Yu, N. J., and Young, D. P., "GMRES Acceleration of Computational Fluid Dynamics Codes," AIAA Paper 85-1494, July 1985.

${ }^{13}$ Meijerink, J. A., and van der Vorst, H. A., "Guidelines for the Usage of Incomplete Decompositions in Solving Sets of Linear Equations as They Occur in Practical Problems," Journal of Computational Physics, Vol. 44. No. 1, 1981, pp. 134-155

${ }^{14}$ Chorin A J. "A Numerical Method for Solving Incompressible Viscous Flow Problems," Journal of Computational Physics, Vol. 2, No. 1, 1967, pp. 12-26.

${ }^{15}$ Roe, P. L., "Approximate Riemann Solvers, Parameter Vectors, and Difference Schemes," Journal of Computational Physics, Vol. 43, No. 2. 1981, pp. 357-372.

${ }^{16}$ Barth, T. J., "Analysis of Implicit Local Linearization Techniques for Upwind and TVD Algorithms," AIAA Paper 87-0595, Jan. 1987.

${ }^{17}$ Baldwin, B., and Barth, T., "A One-Equation Turbulence Transport Model for High Reynolds Number Wall-Bounded Flows," NASA TM102847, Aug. 1990.

${ }^{18}$ Armaly, B. F., Durst, F., Periera, J. C. F., and Schönung, B., "Experimental and Theoretical Investigation of Backward-Facing Step Flow." Journal of Fluid Mechanics, Vol. 127, Feb. 1983, pp. 473-496.

${ }^{19}$ Coles, D., and Wadcock, A. J., "Flying-Hot-Wire Study of Flow Past a NACA 4412 Airfoil at Maximum Lift," AIAA Journal, Vol. 17, No. 4, 1979. pp. 321-329.

${ }^{20}$ Rogers, S. E., Wiltberger, N. L., and Kwak, D., "Efficient Simulation of Incompressible Viscous Flow over Single and Multi-Element Airfoils." AIAA Paper 92-0405, Jan. 1992; see also Journal of Aircraft, Vol. 30. No. 5 , 1993, pp. 736-743.

${ }^{21}$ Chin, V., Peters, D.. Spaid, F., and McGhee, R., "Flowfield Measurements about a Multi-Element Airfoil at High Reynolds Numbers," AIAA Paper 93-3137, July 1993. 